\title{
El desempleo juvenil y la deserción en la Universidad Nacional, sede Bogotá
}

\section{Youth Unemployment and University Dropout at the Universidad Nacional de Colombia, Bogotá Campus}

María Paula Bermúdez Pedraza, Juan Diego Camelo Plazas, Sebastián García Mazo, Daniel Leonardo Garzón Franco, Federico Mediorreal Gutiérrez, Juan Pablo Torres Clavijo ${ }^{1}$

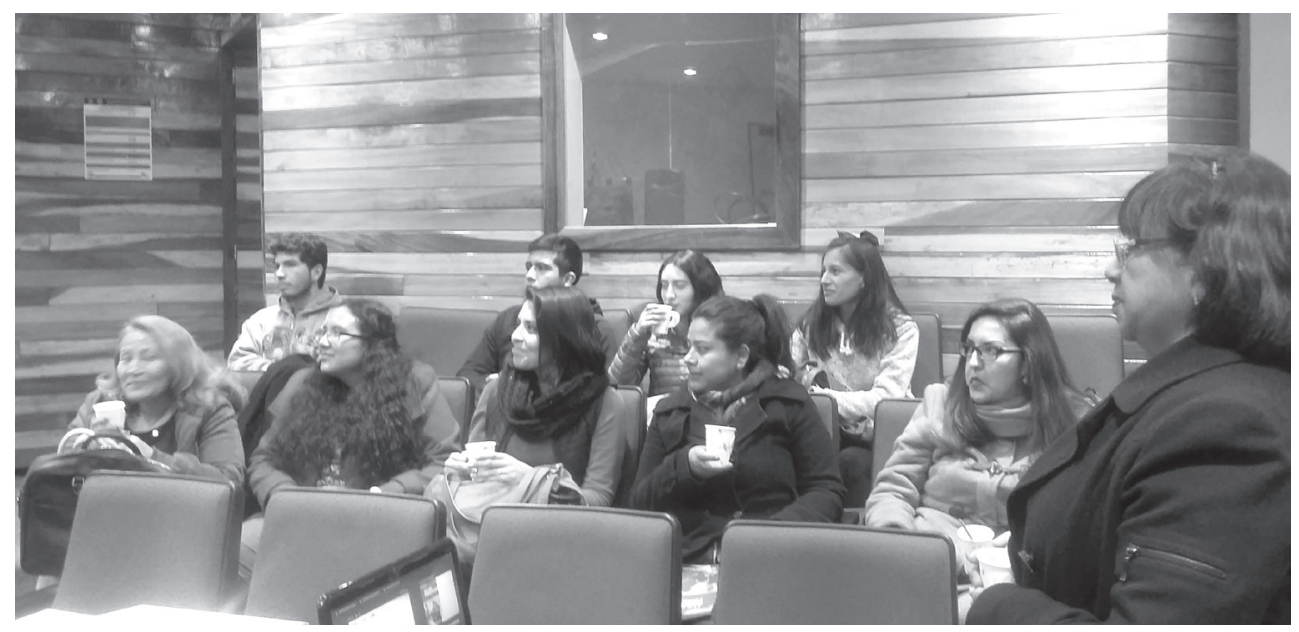

\section{Resumen}

El desempleo juvenil como la deserción estudiantil universitaria son fenómenos que han representado un reto para la sociedad colombiana, los cuales llaman la atención del gobierno nacional a la hora de desarrollar políticas que ofrezcan un panorama estable, lleno de oportunidades para el desenvolvimiento de las futuras generaciones. La presente investigación se llevó a cabo mediante la realización de un estudio entre los estudiantes de pregrado de la Universidad Nacional de Colombia, sede Bogotá, con el fin de establecer y comprender la relación existente entre estos dos fenómenos.

Citar este artículo como: Bermúdez Pedraza, M.P., Camelo Plazas, J. D., García Mazo, S., Garzón Franco, D. L., Mediorreal Gutiérrez, F. y Torres Clavijo, J. P. (2018). El desempleo juvenil y la deserción en la Universidad Nacional, sede Bogotá. Revista Papeles, 10(20), 53-66.

Fecha de recibido: agosto 15 de 2018 - Fecha de aceptación: noviembre 5 de 2018

Estudiantes del curso de Metodología de la Investigación I de la Universidad Nacional de Colombia, sede Bogotá, durante el periodo 2018-II dirigidos por el profesor Enrique Ferrer-Corredor. Correos electrónicos: María Paula Bermúdez Pedraza (mabermudezp@unal.edu.co), Juan Diego Camelo Plazas (jcamelo@unal.edu.co), Sebastián García Mazo (sebgarciama@unal.edu.co), Daniel Leonardo Garzón Franco (dgarzonf@unal.edu.co), Federico Mediorreal Gutiérrez (fmediorreal@unal.edu.co), Juan Pablo Torres Clavijo (jptorrecl@unal.edu.co). 
Palabras clave: desempleo juvenil, deserción universitaria, dependencia e independencia económica, disponibilidad económica.

\begin{abstract}
Youth unemployment and university dropout are the phenomena that represent a challenge to Colombian society, which call the national government's attention when developing policies to provide a stable outlook of the opportunities for future generations. The present research was carried out by making a study among college students of the Universidad Nacional de Colombia, Bogotá Campus, in order to figure out and understand the relationship between these two phenomena.
\end{abstract}

Keywords: youth unemployment, university dropout, economic dependence, economical autonomy and economic availability.

\title{
Introducción
}

En Colombia una de las principales problemáticas respecto a la educación superior es la alta tasa de deserción que se presenta en las IES (Instituciones de Educación Superior). Según la Asociación Colombiana de Universidades (Ascun), esta problemática social afectó al 47 $\%$ de los estudiantes en el año 2018, la más alta en Latinoamérica y superando el promedio de la región, que se calcula en el $37 \%$. Según datos publicados en la revista Semana, se estima que de cada 10 jóvenes 3 abandonan completamente sus estudios; además, 4 de los restantes cambian de programa académico o no logran graduarse después de 14 semestres, lo cual lleva a la pregunta: ¿por qué la deserción en Colombia es tan alta?

Generalmente, se atribuye el hecho de que un estudiante tome la decisión de desertar a problemas personales y familiares, bajo rendimiento académico, falta de apoyo por parte de las IES y carencia de disponibilidad económica, entre otras (Castaño, Gallón, Gómez y Vásquez (2004) y Salcedo (2010). Otro problema que sufren los jóvenes del país es el desempleo, el cual en el año 2018 fue aproximadamente del $26.5 \%$, según cifras del DANE, lo cual indica que a la juventud colombiana le cuesta acceder a empleos. Además de que se enfrentan a condiciones laborales desfavorables que dificultan su permanencia $y$ desarrollo dentro del mercado laboral, como bajos salarios, horarios inflexibles.

Con el fin de identificar la relación existente entre estas dos problemáticas en un contexto real, se decidió realizar este proyecto de investigación en el cual se analiza cómo se presenta la deserción en la Universidad Nacional de Colombia (UN), sede Bogotá, mediante el estudio de las condiciones socioeconómicas de los educandos entre primer y quinto semestre de siete programas académicos de esta institución y de tal manera determinar la incidencia del desempleo juvenil en la deserción estudiantil.

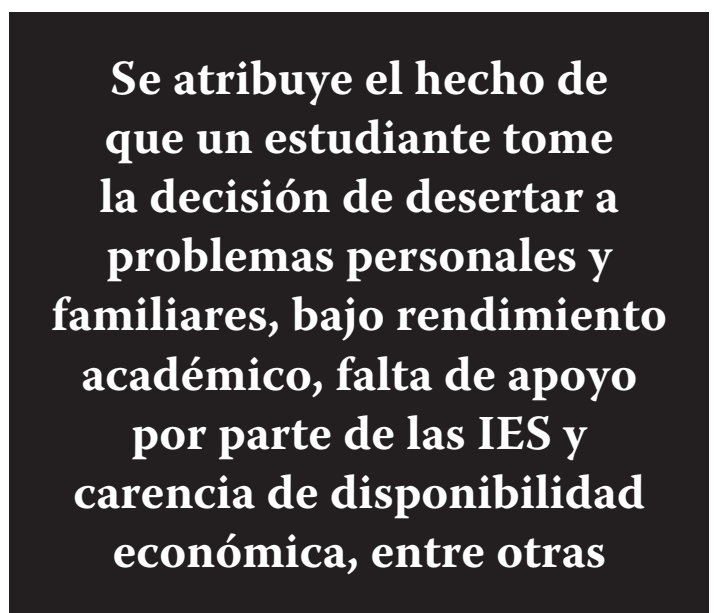




\section{Objetivos}

\section{Objetivo general}

Identificar la relación existente entre el desempleo juvenil y la tasa de deserción en estudiantes que son dependientes e independientes económicamente y que hacen parte de los programas de pregrado de Economía, Fonoaudiología, Ingeniería Agrícola, Ingeniería Civil, Medicina, Música Instrumental y Sociología, matriculados entre primer y quinto semestre en la UN, sede Bogotá.

\section{Objetivos específicos}

Contrastar los resultados de las carreras seleccionadas para así detallar los factores que motivan la deserción y su posible relación con el desempleo juvenil.

Calcular porcentajes y probabilidades de causas de deserción, ingresos por familia, dependencia económica y estrato socioeconómico con el fin de analizar cómo estas variables influencian la relación existente entre desempleo y deserción universitaria.

\section{Marco teórico}

Entiéndase en primer lugar por desempleo juvenil como aquella situación en la que se encuentran los jóvenes cuyo rango de edad está entre los 14 y 28 años (el rango de edad es el establecido en la ley 1622 de 2013), quienes tienen la disposición para obtener un empleo, y que a pesar de ello no están empleados (Arango y Posada, 2001).

Por otra parte, se define deserción estudiantil universitaria como "la disolución del vínculo que se estipula a través de la matrícula académica por cualquier causa ya sea por parte del estudiante o la universidad y tiene graves efectos para las dos partes" (Patiño y Cardona, 2004).

Distintos autores han abordado los fenómenos de deserción estudiantil y desempleo juvenil para intentar comprender sus causas, consecuencias y las características que hacen a los jóvenes más propensos a sufrir de los mismos.

\section{Desempleo juvenil}

El desempleo juvenil se configura como un mecanismo de exclusión social, por lo que tiene un gran impacto en la vida de los jóvenes tanto en su presente como en su futuro. En el corto plazo este fenómeno significa la degradación del capital humano, incapacidad de generar recursos para satisfacer las necesidades e incertidumbre económica (De La Hoz et al., Quejada y Yánez, 2012 y Mora, Caicedo y González, 2017). La falta de empleos de tiempo completo para jóvenes genera altos niveles de rotación laboral. Esto, junto con la necesidad de obtener recursos, hace que los jóvenes se vean forzados a aceptar trabajos en condiciones laborales precarias y mal remuneradas, incentivados a trabajar en la informalidad laboral (Pedraza, 2008 y Yarce, 2000).

Cabe mencionar que el desempleo juvenil no solo tiene implicaciones en el presente de los jóvenes sino también en su futuro laboral, el cual se ve afectado de diferentes maneras debido a la exposición a periodos prolongados de desempleo a edad temprana. El conjunto de estos jóvenes puede llegar a experimentar periodos largos de desempleo en el futuro y restricciones en la generación de ingresos, por lo que el desempleo juvenil llega a dejar cicatrices en los salarios hasta 20 años después de la exposición al mismo (Gregg y Tominey, 2004 y De la Hoz et al., 2012). Adicionalmente, parece haber una relación entre el desempleo juvenil y la motivación a demandar educación. Los jóvenes que afrontan el desempleo 
a temprana edad suelen sentirse frustrados, por lo cual demandan menores niveles de educación (Levin, 1983). De esta manera, el desempleo juvenil afecta el nivel educativo de los jóvenes.

Varios autores estudiados coinciden que algunas de las causas del desempleo juvenil están estrechamente relacionadas al desenvolvimiento económico del país y su estabilidad macroeconómica. La desaceleración económica tiene como consecuencia una reducción en la tasa de creación de empleo, lo cual aumenta las disparidades entre la demanda y la oferta laboral. Estas disparidades afectan principalmente a los jóvenes, pues los empleadores tienen marcadas preferencias por contratar empleados adultos, pues en países como Colombia, en los que está establecido el salario mínimo, los empleadores consideran que los jóvenes no están lo suficientemente cualificados para merecer el pago del mismo, por lo cual no se sienten motivados a contratarlos (Yarce, 2000 y De la Hoz et al., 2012).

Otra de las posibles causas más aludidas por autores como Pedraza (2008), Levin (1983) y García (1987) son las fallas en el sistema educativo. Suele sostenerse que este no hace a los jóvenes lo suficientemente cualificados para los trabajos del presente, lo cual los hace menos preferidos a los trabajadores adultos. Además de la falta de cualificación, los jóvenes se ven expuestos prematuramente al mercado laboral

\section{La falta de empleos de tiempo} completo para jóvenes genera altos niveles de rotación laboral. Esto, junto con la necesidad de obtener recursos, hace que los jóvenes se vean forzados a aceptar trabajos en condiciones laborales precarias y mal remuneradas, incentivados a trabajar en la informalidad laboral. sin tener conocimiento de cómo desenvolverse en el mismo, pues el sistema educativo no les provee las herramientas ni los induce a la integración a sectores gremiales y sindicales.

Algunos autores aluden a la dinámica poblacional como causa de desempleo juvenil, argumentando que debido a las presiones demográficas el ritmo en el que ingresan los jóvenes al mercado laboral es mayor al que este puede absorberlos. Sin embargo, este factor no puede ser visto como determinante a la hora de hablar de desempleo juvenil.

\section{Deserción estudiantil}

Las causas de este fenómeno son frecuentemente agrupadas en cuatro categorías: individuales, académicas, institucionales y socioeconómicas (Castaño, Gallón, Gómez y Vásquez, 2004). Las individuales surgen cuando el estudiante deserta por causas motivacionales o psicológicas; en las académicas la deserción se da principalmente por el bajo rendimiento académico; en las institucionales se da por factores asociados con la institución de educación superior (IES): se consideran la falta de recursos, defectos de organización, superpoblación universitaria, deficiencias docentes y falta de apoyo a los estudiantes cuando estos lo requieren; y en las socioeconómicas se presenta cuando la familia del estudiante no tiene los suficientes ingresos, o porque este mismo se encuentra desempleado y de ello depende la subsistencia en la carrera universitaria (Salcedo, 2010).

Existen múltiples formas de clasificar la deserción, cada una tomando en cuenta un determinado elemento. Por ejemplo, al estudiar el espacio en el cual se produce la deserción se puede compilar en interna (cambio de programa académico), institucional (cambio a otra IES) y total (abandonar el sistema educativo). Otra forma de catalogarla toma en cuenta el momento en el cual ocurre, distinguiendo la deserción precoz (antes de empezar la carrera), deserción temprana (entre primer y 
cuarto semestre), deserción (después de quinto semestre) y deserción total (la suma de las anteriores), entre otros parámetros (Castaño et al., 2004).

Este fenómeno, a pesar de que se ha venido presentando desde hace tiempo, en principio ha llamado la atención del gobierno nacional, el cual, mediante una serie de estrategias, entre las cuales se puede incluir el Sistema de
Prevención y Atención de la Deserción en las Instituciones de Educación Superior (SPADIES), busca reducir la tasa de deserción y sus consecuencias, consideradas desfavorables para toda la sociedad. La más importante de ellas es el aspecto económico, que se ve perjudicado por la escasez de profesionales que impulsen el desarrollo económico del país (Castaño et al., 2004).

\section{Ruta metodológica}

Con el fin de garantizar la confiabilidad y pertinencia de esta investigación se realizó una serie de actividades que marcaron la pauta. En primer lugar, se delimitó el tema y objetivo del presente proyecto. Una vez estos fueron establecidos se escogieron siete carreras de la UN, sede Bogotá, mediante unos parámetros previamente establecidos y relacionados con la tasa de deserción que presentan las mismas. Posterior a la selección de carreras a estudiar se realizó recolección de datos aplicando trabajo de campo, para lo cual se diseñó una encuesta formada por once preguntas, cuyo fin fue definir la probabilidad de que los estudiantes de Economía, Fonoaudiología, Ingeniería Agrícola, Ingeniería Civil, Música Instrumental, Medicina y Sociología matriculados entre primer y quinto semestre deserten debido a la presión ejercida por la poca disponibilidad económica a la hora de continuar sus estudios universitarios, y cómo esta poca disponibilidad está relacionada con la actual crisis del empleo juvenil. Para la obtención de cifras relacionadas al tema se recurrió a tres fuentes: a la oficina de planeación y estadística de la Universidad Nacional, sede Bogotá, para recopilar datos de la institución y, por tanto también a los programas de pregrado analizados. Las cifras sobre deserción estudiantil universitaria a nivel nacional fueron aportadas por el Sistema Para la Prevención y Análisis de la Deserción en las Instituciones de Educación Superior (SPADIES). Adicionalmente, los datos obtenidos referentes al desempleo juvenil fueron recopilados del Departamento Administrativo Nacional de Estadística (DANE). Varias de las cifras recopiladas fueron contrastadas entre sí y con los datos obtenidos en el trabajo de campo para llegar a conclusiones respecto a la investigación.

\section{Análisis de los resultados}

Basados en los datos suministrados por la oficina de planeación y estadística de la Universidad Nacional de Colombia, sede Bogotá, catalogamos las carreras seleccionadas según sus niveles de deserción de la siguiente manera: dos programas de pregrado con deserción alta, Fonoaudiología e Ingeniería Agrícola; tres de deserción media, Economía, Sociología e Ingeniería Civil; dos de deserción baja, Medicina y Música Instrumental.
La oficina de planeación y estadística cuenta con resultados sobre deserción por periodos desde 2013-1 hasta el 2016-1. La tabla 1 recoge los datos sobre las tasas de deserción por periodo del último informado (2016-1), la tasa máxima y la tasa mínima con los periodos en los que se presentaron, correspondientes a los programas de pregrado seleccionados en la UN, sede Bogotá. 
Tabla 1. Sobre las tasas de deserción promedio de las carreras consideradas

\begin{tabular}{|c|c|c|c|}
\hline Carrera / Ítem & $\begin{array}{c}\text { Tasa de desercción en } \\
\mathbf{2 0 1 6 - 1}\end{array}$ & $\begin{array}{c}\text { Máxima tasa de } \\
\text { desercción / Periodo }\end{array}$ & $\begin{array}{c}\text { Mínima tasa de } \\
\text { desercción / Periodo }\end{array}$ \\
\hline Fonoaudiología & $12,7 \%$ & $22,0 \% / 2014-1$ & $5,8 \% / 2014-2$ \\
\hline Ingeniería agrícola & $11,4 \%$ & $20,6 \% / 2013-2$ & $11,4 \% / 2016-1$ \\
\hline Economía & $5,9 \%$ & $9,0 \% / 20132$ & $4,5 \% / 20152$ \\
\hline sociológica & $5,5 \%$ & $10,6 \% / 2013-1$ & $5,1 \% / 2015-2$ \\
\hline Ingeniería civil & $4,4 \%$ & $7,5 \% / 2014-2$ & $4,4 \%-2016-1$ \\
\hline Medicina & $1,6 \%$ & $2,8 \% / 2014-1$ & $1,6 \% / 2014-2$ y 2016-1 \\
\hline Música instrumental & $1,1 \%$ & $6,9 \% / 2013-2$ & $1,1 \%-2016-1$ \\
\hline
\end{tabular}

Fuente: elaboración propia ${ }^{2}$

En la tabla 1 se observa que la tasa de deserción por periodo de las carreras seleccionadas ha tendido a disminuir con el tiempo. Este mismo comportamiento se repite para todos los demás programas de pregrado de la UN, sede Bogotá; pues según la oficina de planeación la deserción por periodo promedio ha disminuido, pasando de estar en $8,3 \%$ en el periodo 2013-1 a 5,6 \% en 2016-1.

Contrastando la tasa de deserción por periodo de la UN, sede Bogotá, 2016-1 (5,6 \%), con la tasa de deserción por periodo nacional para el año 2016 (9,0 \%) presentada por el Ministerio de Educación Nacional (2017), se evidencia que la UN, sede Bogotá, presenta una menor tasa de deserción por periodo que el promedio nacional.

Al trasladarse al trabajo de campo, enmarcado por la encuesta realizada a los estudiantes de la UN, sede Bogotá, de las carreras seleccionadas, se llega a varias conclusiones referentes a sus situaciones con sus responsables socioeconómicos, el estado laboral de los estudiantes, los ingresos de sus hogares y la importancia relativa que le dan a determinados factores a la hora de considerar la posibilidad de desertar.

Como primer hallazgo, los estudiantes de los programas encuestados son en una gran

2 Nota: datos obtenidos de la Oficina de planeación y estadística de la Universidad Nacional de Colombia, sede Bogotá. mayoría totalmente dependientes de sus responsables socioeconómicos, aproximadamente el 67 \%. La segunda clasificación más frecuente es la sostenibilidad mixta con un 29 $\%$, es decir que los gastos del estudiante son cubiertos, una parte, por sus responsables socioeconómicos y, otra, parte por él mismo. Y, por último, total independencia económica, apenas un $4 \%$ :

A pesar de que existe un porcentaje significativo de estudiantes cuya sostenibilidad es mixta, los ingresos que generan parecen ser insuficientes para cubrir el pago de sus matrículas, pues en todos los programas de pregrado seleccionados se observa que la totalidad del pago de la matrícula recae en un gran

Gráfico 1. Situación socioeconómica del total de estudiantes encuestados en la Universidad Nacional, sede Bogotá.

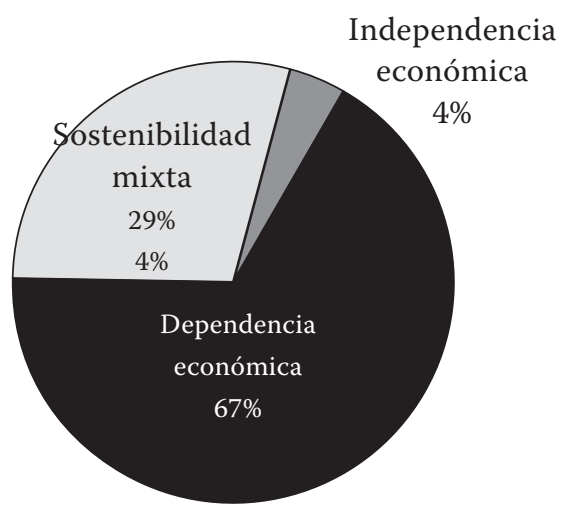

Fuente: elaboración propia 
porcentaje $(69,7 \%)$ sobre los responsables socioeconómicos del estudiante.

Continuando con el análisis de las variables, en cuanto a los ingresos de los estudiantes y sus hogares, la encuesta permite hacerse una idea de cómo se comporta esta variable en las distintas carreras, pues se tiene información sobre el estado laboral de los padres, su estrato socioeconómico y el costo de sus matrículas, teniendo en cuenta que en el UN el costo de la matrícula depende de la situación socioeconómica reportada por el estudiante. Respecto al promedio del estrato socioeconómico, los mayores promedios se presentan en las carreras de Medicina $(3,44)$, Ingeniería Civil $(3,36)$ y Economía (3,2) respectivamente; además, según los resultados obtenidos, se evidencia que dichas carreras tienden a tener matrículas más elevadas que el resto de las encuestadas. Entonces, debido a los resultados obtenidos se podría decir que los estudiantes de las tres carreras anteriormente mencionadas tienen una mayor estabilidad económica que los estudiantes de los demás programas de pregrado considerados, teniendo en cuenta que los puntajes requeridos para ser admitidos a las carreras de Medicina $(760,5)$, Ingeniería Civil $(691,8)$ y Economía $(672,6)$, en este orden, son los tres más altos de los demás programas a considerar (tomando como referencia el periodo 2018-1 con base en el último estudiante admitido).

Un caso contrario se evidencia en los estudiantes pertenecientes a los programas de Ingeniería Agrícola, Fonoaudiología, Música Instrumental y Sociología. Estos presentan los promedios de estrato socioeconómico más bajos de las carreras encuestadas: Ingeniería Agrícola con 2,68, Fonoaudiología con 2,8, Música Instrumental con 2,96 y Sociología con 3,08; además, el costo de la matrícula tiende a ser menor que las demás carreras consideradas. Este resultado es interesante, pues los menores puntajes requeridos en nuestro periodo de referencia (2018-1 y con base al último estudiante admitido) para ingresar a las carreras seleccionadas fueron de Fonoaudiología $(620,0)$, Ingeniería Agrícola $(638,6)$ y Sociología $(645,8)$ respectivamente. Los puntajes requeridos para ingresar a Música instrumental no pueden ser comparados con los de las demás carreras, pues esta carrera presenta particularidades en el proceso de admisión como una prueba específica para la misma. Según el comportamiento anteriormente descrito, podría quizás haber una relación entre ingresos del hogar del estudiante y el programa de pregrado al que el estudiante fue admitido, teniendo en cuenta el puntaje requerido para ingresar al mismo.

Tabla 2. Estratos promedios y puntaje requerido para ingresar a los programas de pregrado encuestados de la Universidad Nacional de Colombia, sede Bogotá.

\begin{tabular}{|c|c|c|}
\hline $\begin{array}{c}\text { Carrera / } \\
\text { criterio }\end{array}$ & $\begin{array}{c}\text { Estrato socioeconómico } \\
\text { promedio }\end{array}$ & $\begin{array}{c}\text { Puntaje último admitido } \\
\text { para 2018 }\end{array}$ \\
\hline Medicina & 3,44 & 760,5 \\
\hline Ingeniería civil & 3,36 & 691,8 \\
\hline Economía & 3,2 & 672,6 \\
\hline Sociología & 3,08 & 645,8 \\
\hline Ingeniería agrícola & 2,68 & 638,6 \\
\hline Fonoaudiología & 2,8 & 620,0 \\
\hline Música instrumental & 2,96 & $*$ \\
\hline
\end{tabular}

Fuente: elaboración propia ${ }^{3}$

3 Nota: el puntaje de Música Instrumental no puede ser comparado con las demás carreras, pues esta presenta particularidades en su proceso de admisión. 
La tabla 3 ofrece cifras con respecto al estado laboral de los estudiantes de las carreras seleccionadas: menos de la mitad de los estudiantes no están empleados, pero tampoco se encuentran buscando empleo. Esto quiere decir que algo más de la mitad, el 53,71\% de los estudiantes, necesita acceder al mercado laboral. Como puede observarse, este 53,71\% de estudiantes que necesitan empleo se compone de la siguiente manera: $0,57 \%$ empleado tiempo completo, $33,14 \%$ tiempo parcial y 20 $\%$ desempleado. Es evidente entonces que los estudiantes de la UN, sede Bogotá, tienen una probabilidad mucho más elevada de terminar aceptando un empleo de tiempo parcial que uno de tiempo completo. Respecto a la tasa de desempleo calculada en nuestra encuesta, podemos concluir que no varía significativamente frente a la tasa de desempleo juvenil en Colombia, pues para el último trimestre del que se tiene información, octubre-diciembre de 2018, la tasa de desempleo en jóvenes fue de 16,4 \% (DANE, 2019), mientras que, como se mencionó anteriormente, nuestra estimación para la UN, sede Bogotá, fue $20 \%$.

Analizando el porcentaje de estudiantes que necesitan acceder al mercado laboral $(57,71$

Tabla 3. Estado laboral de los estudiantes de los programas de pregrado encuestados de la Universidad Nacional de Colombia, sede Bogotá.

\begin{tabular}{|l|c|c|}
\hline \multicolumn{1}{|c|}{$\begin{array}{c}\text { Estado } \\
\text { Laboral }\end{array}$} & $\begin{array}{c}\text { No. de } \\
\text { personas }\end{array}$ & $\begin{array}{c}\text { \% de } \\
\text { personas }\end{array}$ \\
\hline $\begin{array}{l}\text { A) Empleado tiempo } \\
\text { completo }\end{array}$ & 1 & $0,57 \%$ \\
\hline $\begin{array}{l}\text { B) Empleado tiempo } \\
\text { parcial }\end{array}$ & 58 & $33,14 \%$ \\
\hline $\begin{array}{l}\text { C) No es empleado } \\
\text { y no se encuentra }\end{array}$ & 81 & $46,2 \%$ \\
\hline buscando empleo & & \\
\hline D) Desempleado & 35 & $20 \%$ \\
\hline Total & 175 & 100 \\
\hline
\end{tabular}

Fuente: elaboración propia
$\%$ en promedio para las carreras analizadas) para cada una de las carreras obtenemos los siguientes resultados: Fonoaudiología con $76 \%$, Música Instrumental $64 \%$, Ingeniería Agrícola 60 \%, Economía 52 \%, Sociología 52 $\%$, Medicina $52 \%$ e Ingeniería Civil $20 \%$.

Adicionalmente, se les preguntó a los estudiantes encuestados sobre el peso relativo de determinados factores a la hora de evaluar la opción de desertar de su respectivo programa de pregrado. Estos debían ponderar dichos factores en una escala de 1 a 5 , en la que 1 significaba la menor importancia relativa y 5 la mayor. De los datos obtenidos de todos los programas de pregrado se llega a la conclusión que la importancia relativa de los factores a la hora de tomar la decisión de desertar se presenta en el siguiente orden: falta de disponibilidad económica $(3,005)$, bajo rendimiento académico $(2,754)$, problemas familiares o personales $(2,571)$, cambio de programa o de universidad $(2,32)$ y, por último, bajas expectativas sobre su futuro campo laboral $(1,982)$. Los resultados diseminados por programa de pregrado en relación al factor "falta de disponibilidad económica" se presentan en el gráfico 2, del cual, en relación con lo expuesto anteriormente sobre el ingreso de los estudiantes y el puntaje de admisión, puede notarse que las carreras de Medicina, Ingeniería Civil y Economía son las que menor importancia relativa le dan a este factor, mientras las carreras de Fonoaudiología, Ingeniería Agrícola, Música Instrumental y Sociología le dan una ligera mayor importancia relativa a este factor.

$\mathrm{Al}$ pedirle a los estudiantes que evaluaran la probabilidad de desertar en escala de 1 al 10 , donde 1 es la menor probabilidad y 10 la mayor, se obtuvo un promedio de respuesta de $26,97 \%$ entre todos los programas de pregrado encuestados. De este resultado lo más revelador fueron las respuestas de los estudiantes de Música Instrumental y Medicina, las cuales fueron las que menor probabilidad 
Gráfico 2. Importancia relativa diseminada por carreras del factor "falta de disponibilidad económica" a la hora de plantearse la posibilidad de desertar.

Falta de disponibilidad económica

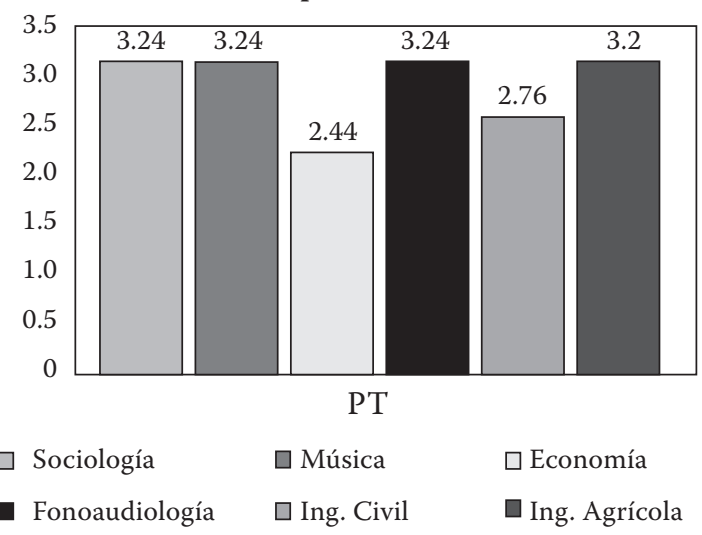

Fuente: elaboración propia. presentaron: Música Instrumental con $21,2 \%$ y Medicina con 22\%. Teniendo en cuenta que Música Instrumental y Medicina son las dos carreras con menores tasas de deserción respectivamente, puede suponerse que estos valoran su cupo por la dificultad que revistió conseguirlo.

Por último, cuando les preguntamos a los estudiantes si actualmente encontraban en el emprendimiento (desde su expresión más básica como la venta de golosinas, hasta formas desarrolladas de emprendimiento como la creación de una empresa propia) una alternativa para financiar sus estudios universitarios, más de la mitad, el 55,43\%, respondió de manera afirmativa.

\section{Discusión}

Durante el análisis de los resultados surgieron varias afirmaciones que se irán sustentando con base a los resultados de la encuesta y la información recopilada de los autores leídos, donde se espera encontrar relaciones y poder responder a nuestra hipótesis principal: el desempleo juvenil es una de las causas en la deserción de los estudiantes universitarios.

Sobre la deserción en universidades públicas surge un contraste con Melguizo, Sánchez y Jaime (2011) y con Barragán y Patiño (2013). Los primeros afirmaron que la universidad pública presenta mayores tasas de deserción debido a la falta de ayuda financiera por parte del gobierno y por la mala organización y estructura de esta misma. Por su parte, Barragán y Patiño (2013) se limitaron a sustentar que no se presenta una diferencia notoria entre la universidad pública y privada en cuanto a deserción. Un resultado al que se llegó a partir de la contratación de los datos suministrados por SPADIES y la oficina de planeación de la Universidad Nacional de Colombia, sede Bogotá, es que las tasas de deserción por periodo en tal universidad son significativamente menores que el promedio nacional; pues mientras que para el periodo 2016-1, la UN, sede Bogotá, presentó tasas del 5,6\%, el promedio de las demás IES correspondió al $9,0 \%$. Este fenómeno puede deberse a que el pago de la matrícula de la UN tiene en cuenta la situación socioeconómica del estudiante, lo que hace que su pago sea "más justo" que en las IES cuyo pago suele estar estandarizado. Además, basados en lo que argumentaron varios de los autores referenciados, existen causas de deserción institucionales, que son propias de la IES. Sobre este aspecto podríamos decir que la UN tiene una adecuada institucionalidad en cuanto a respaldar a sus estudiantes se refiere, pues esta cuenta con distintos apoyos como subsidios de transporte y alimentación, posibilidades de fraccionamiento de matrícula, oportunidades laborales para sus estudiantes, etc. De manera tal que los estudiantes vulnerables económicamente tienen más oportunidades de continuar sus estudios en la UN que en una IES privada. 
Enfocándonos en cifras sobre desempleo, se compara la tasa de desempleo juvenil estimada en nuestra encuesta para los estudiantes de la UN, sede Bogotá, que fue del $20 \%$, con la tasa de desempleo juvenil nacional, que es del $16,4 \%$. Notamos que la primera presenta una tasa ligeramente mayor a la tasa nacional. Este hecho explicaría por qué el emprendimiento en la mencionada IES es tan común, pues 55,43\% de los jóvenes afirman que mediante el mismo sustentan parte de sus estudios universitarios. $\mathrm{Al}$ ser el desempleo ligeramente superior en la UN, sede Bogotá, y debido a las pocas oportunidades que ofrece el mercado laboral para los jóvenes, estos se desplazan hacia distintas formas de generar recursos, viendo el emprendimiento como una opción viable.

Esta investigación enfoca su análisis sobre los factores socioeconómicos que motivan la deserción, pues de esta manera se articula a la problemática del desempleo juvenil. En relación con varios de los autores estudiados y con los resultados obtenidos se puede establecer una relación entre la escasez de recursos económicos en el hogar de los jóvenes y la necesidad de estos de buscar un empleo. De esta manera, de acuerdo con los resultados obtenidos en la encuesta notamos que las diferencias marginales en el estrato económico y el costo de la matrícula (que como se explicó anteriormente reflejan el nivel de ingreso del hogar) tienen impacto fuerte en la probabilidad de desertar de los estudiantes, pues cabe recordar que los programas de pregrado catalogados como de alta deserción, Fonoaudiología e Ingeniería Agrícola, son aquellos que según los datos recopilados presentan menores ingresos. Este hecho se refuerza con los datos obtenidos sobre el factor al que los estudiantes le dan mayor importancia relativa a la hora de tomar la decisión de desertar, "falta de disponibilidad económica". Puede decirse que los estudiantes de menores ingresos tienen mayores necesidades de acceder al mercado laboral, en donde no consiguen empleos de calidad que les faciliten dar continuidad a sus carreras, de tal manera el desempleo juvenil se configura como causa de deserción e influye más en estudiantes de bajos recursos.

Respecto a la población universitaria que necesita acceder al mercado laboral se puede llegar a varias conclusiones. Recordando que el promedio de jóvenes que necesitan empleo para las carreras encuestadas fue de $53,71 \%$, las carreras cuyos datos están por encima de la media son: Fonoaudiología con 76 \%, Música Instrumental 64 \% e Ingeniería agrícola $60 \%$. Por ende, teniendo en cuenta lo anteriormente planteado en la presente discusión, puede concluirse que los estudiantes de Fonoaudiología e Ingeniería Agrícola tienen mayores necesidades de acceder al mercado laboral, pues los hogares de dichos estudiantes tienen menores recursos que los de las demás carreras analizadas. De esta manera la falta de recursos obliga a los estudiantes a buscar soluciones en el mercado laboral, el cual no les brinda condiciones óptimas para compaginar sus estudios con un empleo, de manera tal que este fenómeno afecta sus probabilidades de permanencia en la universidad, y es en parte por esto que estas carreras se ubican entre las de alta deserción (véase tabla 1). En cuanto a Música Instrumental se presentan varias particularidades que hacen invalida la tesis previamente planteada para este programa de pregrado, pues Música Instrumental presenta muy bajos niveles de desempleo en comparación a las otras dos carreras previamente mencionadas, de manera tal que la mayoría del $64 \%$ de estudiantes que necesitan acceder al mercado laboral tiene un empleo de tiempo parcial. Indagando con estudiantes sobre el porqué de este fenómeno llegamos a la conclusión de que su ámbito laboral les facilita encontrar un empleo que compagina con su carrera. En este marco, las asesorías musicales se muestran como una opción con alta demanda que permite que estos estudiantes se inserten al mercado laboral sin traumatismos y desarrollen con normalidad su programa de pregrado. Por lo anteriormente mencionado, 
podría decirse que el desempleo juvenil no afecta a Música Instrumental de la manera que lo hace con Fonoaudiología e Ingeniería Agrícola, de manera que Música Instrumental se coloca como la carrera de menor deserción en la UN, sede Bogotá.

Analizando detalladamente los datos obtenidos sobre los estudiantes que necesitan acceder al mercado laboral en la UN, sede Bogotá, evidenciamos un aspecto interesante sobre la tasa de ocupación de los jóvenes encuestados, la cual es del $33,71 \%$. La tasa de ocupación se compone por un 33,14 \% de jóvenes empleados a tiempo parcial frente a un $0,57 \%$ empleados a tiempo completo. La enorme diferencia entre el porcentaje de jóvenes empleados a tiempo parcial frente a aquellos empleados a tiempo completo puede deberse a dos razones: la primera, es que la UN, sede Bogotá, no ofrece horarios flexibles (estrictamente nocturnos o diurnos), de tal manera que a los jóvenes les es extremadamente difícil compaginar sus estudios con un empleo de tiempo completo. Una segunda razón estaría en sintonía con lo expuesto por De La Hoz et al., (2012), existe una carencia de empleos de tiempo completo para los jóvenes, pues estos presentan mayor rotación laboral que los empleados adultos, por esta razón a los jóvenes les cuesta más encontrar un empleo de tiempo completo.

Con base a lo anterior y recordando que nuestra estimación sobre la tasa de desempleo de los jóvenes en la UN, sede Bogotá es del $20 \%$, la cual estaba ligeramente por encima de la nacional $(16,4 \%)$, puede decirse que este fenómeno se presenta en parte debido a la marcada predilección de los jóvenes de la UN, sede Bogotá, por empleos de tiempo parcial; pues, continuando con lo argumentado por De La Hoz et al., (2012), los jóvenes registran mayores niveles de rotación laboral debido a que el empleo más común entre estos es el empleo de tiempo parcial, el cual los hace más propensos a sufrir un despido.
Sin embargo, los datos recopilados refutan una hipótesis que se planteó al principio de la investigación. Se pensaba que en aquellas carreras cuyo puntaje de admisión era bajo los jóvenes iban a dar mayor importancia relativa a los factores "bajas expectativas sobre su futuro campo laboral" y "cambio de programa y/o universidad" a la hora de evaluar su propensión a desertar. Se planteó esta hipótesis bajo el supuesto de que los bajos puntajes requeridos en carreras como Fonoaudiología, Ingeniería Agrícola y Sociología significaban que eran poco demandadas debido a la percepción negativa que generaba su campo laboral. Relacionando lo anterior con el desempleo juvenil, se tenía la idea de que los jóvenes consideran que les será difícil conseguir empleos de calidad en las áreas de estas carreras. Todo lo anteriormente mencionado basado además en la tesis de Barragán y Patiño (2013) y Mora et al., (2017), quienes sugerían que el desempleo juvenil impacta en la deserción, pues los jóvenes no consideran útil estudiar, ya que se enfrentan a una situación desfavorable para ingresar al mercado laboral. Contrastando nuestra hipótesis, los factores "bajas expectativas sobre su futuro campo laboral" y "cambio de programa y/o universidad" presentaron los promedios más bajos y sin diferencias significativas entre carreras, por lo cual se puede concluir que las bajas expectativas de los jóvenes sobre su futuro campo laboral no es un factor determinante a la hora de desertar para ninguna carrera.

La falta de recursos obliga a los estudiantes a buscar soluciones en el mercado laboral, el cual no les brinda condiciones óptimas para compaginar sus estudios con un empleo, de manera tal que este fenómeno afecta sus probabilidades de permanencia en la universidad. 


\section{Conclusiones}

Los datos recopilados durante la investigación nos llevan a concluir que existe una relación bidireccional entre el desempleo juvenil y la deserción estudiantil universitaria. En cuanto a la incidencia de la primera sobre la segunda, notamos que afecta principalmente a los jóvenes de hogares con bajos recursos, debido a que estos tienen la necesidad de buscar una manera para financiar sus gastos, por lo cual deben acceder al mercado laboral, y es entonces que la crisis del empleo juvenil afecta la continuidad educativa de los jóvenes en dos formas similares.

En primera instancia, los jóvenes se enfrentan a un mercado laboral que no los acoge de la manera que estos necesitan; por ende, se ven enfrentados a condiciones laborales precarias y mal remuneradas, las cuales deben aceptar de acuerdo a sus necesidades, es decir, los jóvenes de menores recursos tienen mayores incentivos para aceptar dichas condiciones. De esta manera, dichos empleos con condiciones precarias no compaginan con sus estudios, de manera tal que los jóvenes al tener determinadas preocupaciones laborales no pueden dedicarse a sus respectivos programas de pregrado, viéndose sustancialmente afectado su desempeño y estabilidad emocional, de manera tal que hay una incidencia en su decisión de desertar.

La segunda manera radica en que los jóvenes no encuentran empleo en el mercado laboral y de esta manera no pueden generar recursos económicos que les permitan sustentar su permanencia en la universidad; la falta de disponibilidad económica y las preocupaciones que esta conlleva tendrían entonces incidencia en la deserción estudiantil universitaria.

Ya habiendo argumentado la relación existente de nuestros dos tópicos en una dirección (desempleo juvenil-deserción estudiantil universitaria), se puede analizar el fenómeno en dirección contraria, es decir, la incidencia de la deserción estudiantil universitaria sobre el desempleo juvenil. Basado en varios autores y en las cifras del DANE (2018) notamos que la educación es el motor principal de ascenso social, es decir, entre mayores niveles de educación mayor será la probabilidad de acceder a un empleo calidad. De manera contraria, las capacidades no adquiridas mediante la educación aumentan la probabilidad de estar desempleado, de tener bajos salarios y un empleo de baja calidad. Por lo tanto, cuando un joven deserta de la universidad está perdiendo la oportunidad de generar capital humano que le facilite conseguir un empleo, de manera tal que el haber desertado afecta su futuro laboral.

Con base en lo anterior llegamos a la conclusión central de que existe una correlación entre las causas de desempleo juvenil y deserción estudiantil.

La relación causal entre desempleo juvenil y deserción estudiantil es clara. Si el estudiante tiene una familia de pocos ingresos este se ve obligado a buscar empleo, durante el periodo que busca el trabajo este estudiante es parte del grupo de personas en desempleo. Buscar un trabajo aumenta el nivel de deserción debido a que el estudiante se preocupa más en subsistir económicamente dejando de lado el mantener un buen nivel académico.

\section{Los jóvenes se ven enfrentados a condiciones laborales precarias y mal} remuneradas, las cuales deben aceptar de acuerdo a sus necesidades, es decir, los jóvenes de menores recursos tienen mayores incentivos para aceptar dichas condiciones. 


\section{Referencias}

Arango. L. \& Posada. C. (2001) El desempleo en Colombia. Banco de la República. Recuperado de: http://www.banrep.gov.co/docum/ ftp/borra265pdf.pdf

Arango. M. \& Ramírez. D. (2007) Análisis desde la teoría motivacional de las causas de ingreso y deserción del estudiante universitario. Scientia et Technica Año XIII, No 37, diciembre de 2007. Universidad Tecnológica de Pereira.

Barragán. D \& Patiño. L (2013) "Elementos para la comprensión del fenómeno de la deserción universitaria en Colombia. Más allá de las mediciones". Cuadernos Latinoamericanos de Administración, vol. IX, núm. 16, pp. 55-66

Beyer, H. (1998). "Desempleo juvenil o un problema de deserción escolar?” (No. 277). Centro de Estudios Públicos

Bolance, C et al. (2012). "Deserción y graduación estudiantil universitaria: una aplicación de los modelos de supervivencia" Universia. vol. III. núm. 6.

Castaño. E., Gallón. S, Gómez. K., \& Vásquez. J. (2004) Deserción estudiantil universitaria: una aplicación de modelos de duración. Lecturas de Economía, 60, pp. 39-65.

DANE (2018) "Saber para decidir - Sistema nacional de información de demanda laboral". Recuperado de: https://www.dane.gov. co/files/investigaciones/boletines/sinidel/ boletin-sinidel-2018.pdf.

DANE (2019) "Mercado laboral de la Juventud Trimestre octubre - diciembre 2018". Recuperado de: https://www.dane.gov.co/files/ investigaciones/boletines/ech/juventud/ Bol_eje_juventud_oct18_dic18.pdf

De La Hoz, F. J., Quejada, R. \& Yánez, M. (2012). El desempleo juvenil: problema de efectos perpetuos. Revista Latinoamericana de Ciencias Sociales, Niñez y Juventud, 10, (1), pp. 427-439.
Elkin Castaño, Santiago Gallón, Karoll Gómez, Johanna Vásquez (2004). Lect. Econ. No. 60. Medellín, enero - junio 2004, pp. 39-45

García, E. (1987). “Acerca de algunos elementos ideológicos en el debate sobre escolaridad de masas, desempleo juvenil y división del trabajo". Mientras Tanto, No. 30/31, pp. 177-191. Publicado en: Icaria Editorial.

Goicovic Donoso, Igor. (2002). Educación, deserción escolar e integración laboral juvenil. Última década, 10(16), 11-52. https://dx.doi.org/10.4067/S0718-22 362002000100002

International Labour Organization (2012) "La crisis del empleo juvenil: Un llamado a la acción Resolución y conclusiones de la 101. ${ }^{a}$ reunión de la Conferencia Internacional del Trabajo, Ginebra, 2012". ILO. Recuperado de: https://www.ilo.org/wcmsp5/groups/pu blic/---ed_norm/--relconf/documents/ meetingdocument/wcms_187080.pdf.

La mayoría de universitarios no terminan la carrera que empiezan (17 de septiembre de 2017) Revista Semana. Recuperado de: https://www.semana.com/educacion/ar ticulo/desercion-escolar-y-baja-tasas-degraduacion-en-colombia/540691

Levin. H. (1983). "Youth Unemployment and Its Educational Consequences”. Educational Evaluation and Policy Analysis, Vol. 5, No. 2, pp. 231-247. Publicado por: American Educational Research Association.

Lynch, L. (1983). "Job Search and Youth Unemployment". Oxford Economic Papers, New Series, Vol. 35, Supplement: The Causes of Unemployment, pp. 271-282. Publicado por: Oxford University Press.

Macías. S \& Reyes. A. (2004). "MIGRACIÓN LABORAL Y DESERCIÓN EDUCATIVA". Canadian Journal of Latin American and Caribbean Studies, Vol. 29, No. 57/58, International Migration in the Americas, pp. 173-202. Publicado en: Taylor \& Francis, 
Ltd. on behalf of Canadian Association of Latin American and Caribbean Studies.

Melguizo. T, Sanchez. F \& Jaime. H. (2011). "The association between financial aid availability and the college dropout rates in Colombia”. Higher Education, Vol. 62, No. 2, pp. 231-247. Publicado por: Springer.

MINISTERIO DE EDUCACIÓN NACIONAL. (2013) "Acuerdo nacional para disminuir la deserción en la educación superior". MEN. recuperado de: https://www.mineducacion.gov.co/sistemasdeinformacion/1735/ articles-254702_archivo_pdf_politicas_estadisticas.pdf.

MINISTERIO DE EDUCACIÓN NACIONAL (2009) "Educación superior a blindarse para enfrentar la crisis económica”. MEN. recuperado de: https://www.mineducacion. gov.co/sistemasdeinformacion/1735/articles-358471_recurso_11.pdf.

MINISTERIO DE EDUCACIÓN NACIONAL (2017). "REPORTE SOBRE DESERCIÓN Y GRADUACIÓN EN EDUCACIÓN SUPERIOR AÑO 2016". MEN. Recuperado: https://www.mineducacion.gov.co/sis temasdeinformacion/1735/articles-3575 49_recurso_5.pdf

Mora. J, Caicedo. C \& González C. (2017). "La duración del desempleo de los jóvenes y los "ninis" en Cali". Revista de economía institucional. Vol. 19, n. 37, pp. 167-184. ISSN 0124-5996. http://dx.doi.org /10.18601/01245996.v19n37.09

Narváez, V. (25 de septiembre de 2018). En Colombia, el $47 \%$ de los alumnos deserta de la universidad. Diario El Heraldo. Recuperado de: https://www.elheraldo.co/barranquilla/en-colombia-el-47-de-los-alumnos-deserta-de-la-universidad-545962

Patiño. L. \& Cardona. A (2012). "Revisión de algunos estudios sobre la deserción estudiantil universitaria en Colombia y Latinoamérica". Theoria, Vol. 21, núm. 1.pp. 9-20.
Pedraza. A. (2008) El mercado laboral de los jóvenes y las jóvenes en Colombia: Realidades y respuestas políticas actuales. Revista Latinoamericana de Ciencias Sociales, Niñez y Juventud. vol. 6, núm. 2. pp. 853-884.

Preocupante radiografía: 4 de cada 10 jóvenes están sin empleo en Colombia (2018). Revista Semana. Recuperado de: https:// www.semana.com/nacion/articulo/ desempleo-juvenil-en-colombia/565565

Rojas, M. (2009). El abandono de los estudios: Deserción y decepción de la juventud. Hologramática. - Facultad de Ciencias Sociales UNLZ, Número 10, V4, pp. 75-94

Salcedo. A. (2010). Deserción universitaria en Colombia. [archivo PDF]. Revista Académica y Virtualidad. Recuperado de: http:// www.alfaguia.org

Sapelli, Claudio, \& Torche, Arístides. (2004). Deserción Escolar y Trabajo Juvenil: ¿Dos Carasde Una Misma Decisión? Cuadernos de economía, 41(123), 173-198. https://dx.doi. org/10.4067/S0717-68212004012300001

Torres, F., \& Zúñiga, J. (2013). Deserción en la educación superior en Colombia durante la primera década del siglo XXI: ¿por qué ha aumentado tanto? In Silva L. (Ed.), La educación superior: Retos y perspectivas (pp. 307-342). Universidad de los Andes, Colombia

Wietse de Vries, Patricia León Arenas, José Francisco Romero Muñoz e Ignacio Hernández Saldaña (2011). Rev. educ. sup vol.40 no.160 México oct./dic. 2011. Recuperado de http://www.scielo.org.mx/ scielo.php?script=sci_arttext\&pid=S01 85-27602011000400002

Yarce. W. (2000) El desempleo estructural y la tasa natural de desempleo. Lecturas de Economía, ISSN-e 0120-2596, N.․ 52, pp. 87-112 